\title{
Sliding Mode Observer Design for a Parabolic PDE in the Presence of Unknown Inputs
}

\author{
Y. Orlova , S. Chakrabarty ${ }^{\text {}}$, D. Zhao ${ }^{\text {, }}$, and S. K. Spurgeon ${ }^{d}$
}

\begin{abstract}
This paper considers observer design for systems modelled by linear partial differential equations (PDEs) of parabolic type which may be subject to unknown inputs. The system is assumed to have only one spatial dimension, over which it is discretised to obtain what is referred to as the lattice system which is a set of linear time invariant (LTI) ordinary differential equations (ODEs) having a canonical Toeplitz-like structure with a specific sparsity pattern. This lattice structure is shown to be particularly appropriate for stepby-step sliding mode observer design which can reconstruct the state estimates at the points of discretisation and estimate the unknown input. Simulation results for both stable and unstable PDEs show that accurate state estimates can be provided at the points of discretisation. An approach to reconstruct the unknown input is demonstrated.
\end{abstract}

Key Words: step-by-step observer; sliding mode; partial differential equation; spatial discretisation; finite time estimation; unknown input estimation

\section{Introduction}

Partial differential equations (PDEs) have been commonly used to model various physical phenomena distributed over time and space. Examples include heat flow, diffusion and waves. Control of systems modelled by PDEs continues to receive attention in the literature [28], [29]. From the point of view of controller implementation, using PDE models has some practical disadvantages. A PDE model is representative of an infinite dimensional system and thus a state feedback controller designed based on the PDE model will require measurements across the entire domain. The advent of micro-electromechanical system (MEMS) technology facilitates this (see [2] for details) but it

${ }^{\mathrm{a} C I C E S E}$, Electronics \& Telecommunications, Ensenada, Mexico; ${ }^{b}$ Department of Electrical Engineering, IIT Roorkee, India; ' College of Chemical Engineering, China University of Petroleum, Qingdao, China; ${ }^{\mathrm{d}}$ Department of Electronic and Electrical Engineering, University College London, UK

This work was supported by EPSRC grant reference number EP/J018295/1, a Chinese Ministry of Education Chiang Jiang International Scholarship and NSFC (61473312). may not be desirable or economically viable for some applications. This motivates work to reconstruct the distributed state of the PDE based on the available measurements at individual points. Such measurements are frequently located at the boundaries. In addition, it is of interest to estimate a disturbance affecting the system at one or more spatial position [11], for example for the purposes of fault detection or condition monitoring.

For ease of exposition, observer design for a linear reaction-advection-diffusion PDE in a scalar spatial dimension is considered. Extension to higher spatial dimensions and other classes of PDEs is possible but the development is rather involved. The method presented here contrasts to many existing PDE-type methods of control/observation of distributed parameter systems (DPSs) rooted in a specific plant equation where nontrivial extensions are required to extend the approach for alternative dynamic system representations. Previously considered methods include the sliding mode approach, backstepping method and active disturbance rejection (see [18], [21], [13], and references therein).

Discretisation of the PDE along the spatial dimension is undertaken to produce an LTI system of 
ODEs with a tridiagonal system matrix. Each diagonal has the same entry throughout its length, apart from the first and last elements of the main diagonal. In a Toeplitz form, each diagonal has the same entries along its length. The lattice system is thus not in Toeplitz form because the first and last elements of the main diagonal are different. For this reason, the system matrix of the lattice system is said to be in a Toeplitz-like form. Sliding mode observer design for systems where the state distribution matrix has lower Hessenberg form is considered. A step-by-step approach to sliding mode observer design as in [3], [19], [12] is particularly appropriate for systems in this lower Hessenberg form. It is demonstrated that the tridiagonal Toeplitz-like form resulting from the spatial discretisation process belongs to the class of systems with lower Hessenberg form and thus the proposed sliding mode observer design can be directly applied to the lattice system. The tridiagonal terms of the resulting Toeplitz-like matrix accumulate the reaction-advectiondiffusion values. The proposed observer design method does not require the state transformation typically used for eliminating the advection term as used in the PDE treatment of a scalar reaction-advection-diffusion PDE (see, e.g., [16, Section 4.7]). This, in any case, is not viable for coupled PDEs of the same type.

The sliding mode approach is well established as a robust approach to both controller and observer design. The technique has received significant attention from the points of view of both theoretical development [26] and practical application [27],[30]. In the finitedimensional setting, sliding mode observers are well established [7],[8],[14],[10],[22]. The step-by-step observer is a particular sliding mode observer generally employed for systems having special structural forms [3],[19],[12]. Such forms typically allow successive state estimation (of one state independent of the others, and offer estimation of the states sequentially). In finite time, all the states are estimated one by one. This approach is known to be powerful when it is required to estimate an unknown disturbance, which can be considered as an unknown input to the system [5], [12]. Specific extensions when the unknown input is unmatched are considered in [31], [32] and [33]. Alternative observer approaches that can be used to estimate unknown inputs are described in [1], [4].

In this work, the observer is designed for ODE systems in the tridiagonal Toeplitz-like form, naturally inherited from the PDE setting and also relevant to the network setting [17]. It utilizes switched injection terms traditionally used in sliding mode controllers and observers, giving them the celebrated property of inherent robustness [25],[9]. Since the approach adopted here is one of spatial discretisation, an observer designed using such a robust method seems an appropriate choice as it can be expected to ensure robustness against the approximation due to discretisation. The contribution is to utilize for the first time a robust step-by-step observer which can easily be designed in an ODE setting for the state and unknown input estimation problem of a corresponding PDE system. This bypasses all methodologies of observer design in the PDE framework, which are limited in their nature as they work for a specific plant and cannot be extended to other dynamic plants. Hence, a design approach which is straightforward, scalable and plant independent has been proposed for observer design of significant classes of PDE systems. The method can be applied to all systems of the form of a linear reaction-advection-diffusion PDE and a similar observer structure could be utilized for network systems described using diffusion-reaction terms.

The paper is structured as follows: Section 2 introduces the class of PDE systems under consideration and defines the boundary and initial conditions. A spatial discretisation of the PDE is performed to yield the tridiagonal Toeplitz-like ODE system. In Section 3, a step-by-step sliding mode observer is developed for a LTI arbitrary finite order ODE system with a lower Hessenberg structure. Conditions for finite time convergence of the state estimates are presented and it is shown that unknown input signals could also be reconstructed. A simulation study is performed with the proposed sliding mode observer for a system in the tridiagonal Toeplitzlike matrix form used to model the lattice system. It is shown that the state estimation errors go to zero in finite time for different orders of the model. Conditions for estimation of the unknown input are also explored. In section 4 , the proposed observer design is applied directly to both a stable and unstable PDE system. The simulation results show that the observer reconstructs the state estimates at the points of discretisation with high accuracy in both cases. Performance in the presence of an unknown input signal is explored. It is shown that good estimates can be obtained at the points of discretisation and that it is possible to determine useful information about the unknown input from analysis of the observer injection signals. In section 5, conclusions are stated and future directions are highlighted. 


\section{Generalized Heat Transfer Model}

Consider a standard space-normalized heat transfer equation in one dimension

$$
T_{t}=k_{1} T_{x x}+k_{2} T+k_{3} T_{x}
$$

where $T_{t}$ is the first partial derivative of $T$ with respect to (w.r.t.) time and $T_{x}$ and $T_{x x}$ are respectively the first and the second order partial derivatives of $T$ w.r.t. space (in the x-direction). Here $T=T(t, x)$ denotes the temperature at a particular time instant $t \geq 0$ and a space point $x \in[0,1]$, the positive parameter $k_{1}$ stands for the diffusivity, and $k_{2}$ and $k_{3}$ for the reaction and advection, respectively. Let the above equation be associated with the following Robin boundary conditions and initial condition.

\section{Boundary Conditions}

$$
\begin{aligned}
T_{x}(0) & =\kappa_{0} T(0, t) \\
T_{x}(1)+\kappa_{1} T(1, t) & =q(t)
\end{aligned}
$$

Initial Conditions

$$
T(0, x)=T_{0}(x)
$$

where the parameters $\kappa_{0}$ and $\kappa_{1}$ are constants. Throughout, the initial distribution $T_{0}(x)$ and heat flux $q(t)$ are assumed to be reasonably smooth functions of their arguments to deal with classical solutions of the boundary-value problem (1)-(3). This assumption is made for a technical reason to ensure [6] that the solutions evolve in the Sobolev state space $H^{2}(0,1)$ of continuous functions with square integrable derivatives such that the Robin boundary conditions (2) are satisfied. The boundary conditions correspond to the case where one end $(x=0)$ is insulated from the surroundings and hence there is no exchange of heat flux at that position. The heat flux $q(t)$ is supplied at the other end but is unknown.

The initial condition gives a profile of the temperature distribution at the various points $x \in[0,1]$ when $t=0$. It is assumed that the temperature at the end $x=0$, i.e., $T(t, 0)$ is known by measurement for all time $t$. Hence the system output is given by $y(t)=$ $T(t, 0)$. For theoretical developments it is necessary that the absolute maximum values of the temperatures at each spatial position as well the absolute maximum value of the input heat flux (at $x=1$ ) are known and bounded. To accommodate the case of unstable PDEs, it is necessary to impose an additional assumption of approximate observability [6] on the PDE system (1). With this assumption, in the case of an unstable PDE, a Luenberger observer can be first designed to ensure boundedness of the system states as required for the design of the step-by-step sliding mode observer.

\subsection{Problem Statement}

The system parameters are assumed to be known and constant and the problem is to estimate the states and the input heat flux from the output measurement $y(t)=T(t, 0)$. This is a state and unknown input observer design problem for the system (1) based only on the output $y(t)=T(t, 0)$.

\subsection{Spatial Discretisation}

The PDE system in (1) is spatially discretized to form a lattice system of $(N+1)$ equidistant divisions along the length of the $x$-dimension with $(N+$ 2) discrete points $i \in\{0,1, \ldots, N+1\}$. Let $T(t)=$ $\left[T_{1}(t) T_{2}(t) \ldots T_{N}(t)\right]^{T}$ be defined as the state vector for the lattice system, giving the temperature at each spatial point of discretisation. The discretisation of $T_{x x}$ at each lattice point can be obtained using the second order central difference scheme [20] as

$$
\left(T_{x x}\right)_{i} \approx \frac{T_{i+1}(t)-2 T_{i}(t)+T_{i-1}(t)}{h^{2}} \forall i=\{1, \ldots, N\}
$$

where $h=\Delta x$ is the unit of spatial discretisation, chosen such a that $(N+1) h=1$ where $N \in \mathbb{N}_{+}$. For the discretisation of $T_{x}$, either the forward difference scheme, the backward difference scheme or the central difference scheme can be employed. Here the central difference scheme which is a second order approximation, with error $h^{2}$, is employed whereas the forward and backward schemes give error of first order. The central difference discretisation scheme gives

$$
\left(T_{x}\right)_{i} \approx \frac{T_{i+1}(t)-T_{i-1}(t)}{2 h} \forall i=\{1, \ldots, N\}
$$

Lemma 1 If the system (1) is spatially discretised to form a lattice system with $(N+1)$ equidistant divisions along its length, the temperatures at the boundary points of the system (1) satisfying the boundary conditions (2) are given as

$$
\begin{aligned}
& T_{0}(t)=\lambda_{0} T_{1}(t) \\
& T_{N+1}(t)=\lambda_{1} T_{N}(t)+h \lambda_{1} q(t)
\end{aligned}
$$

where $\lambda_{i}=\frac{1}{1+\kappa_{i} h}$.

Proof: It is evident that at the boundary $x=0$, only the forward difference scheme can be applied and at the boundary $x=1$, only the backward difference scheme 
can be used. This leads to

$$
\begin{aligned}
T_{x}(0) & =\frac{T_{1}(t)-T_{0}(t)}{h}=\kappa_{0} T_{0}(t) \Rightarrow T_{0}(t)=\lambda_{0} T_{1}(t) \\
T_{x}(1) & =\frac{T_{N+1}(t)-T_{N}(t)}{h}+\kappa_{1} T_{N+1}(t)=q(t) \\
& \Rightarrow T_{N+1}(t)=\lambda_{1} T_{N}(t)+h \lambda_{1} q(t)
\end{aligned}
$$

where $\lambda_{i}=\frac{1}{1+\kappa_{i} h}$.

Lemma 2 The lattice system for the PDE (1) using the central difference scheme for spatial discretisation results in a system of ODEs of the form

$\dot{T}(t)=\left[\begin{array}{cccccc}a_{1} & a_{2} & 0 & 0 & \cdots & 0 \\ a_{3} & a_{4} & a_{2} & 0 & \cdots & 0 \\ 0 & a_{3} & a_{4} & a_{2} & \cdots & 0 \\ \vdots & & & & & \vdots \\ 0 & 0 & 0 & a_{3} & a_{4} & a_{2} \\ 0 & 0 & 0 & 0 & a_{3} & a_{5}\end{array}\right] T(t)+\left[\begin{array}{c}0 \\ \vdots \\ 0 \\ a_{0}\end{array}\right] q(t)$ $y(t)=\left[\begin{array}{llll}1 & 0 & \ldots & 0\end{array}\right] T(t)$

where $a_{i}, \quad i=\{0,5\}$ are related to the system parameters and the discretisation step (Table 1).

Proof: The proof is straightforward and is omitted.

\subsection{Accuracy of the model approximation}

It is important to verify the degree to which the responses of the lattice system and the original PDE system correlate. If an observer is to be designed based upon the lattice system representation, the likely mismatch between the responses of the actual PDE system and the model used for observer design must be quantified. The original PDE system is simulated using the pdepe program in Matlab [15], which is an inbuilt Matlab program to accurately simulate a PDE system. In this paper, all the PDE simulations are computed using pdepe. Simulation parameters are set to the program defaults and system parameters selected as $k_{1}=1, k_{2}=-0.5$ and $k_{3}=-0.5$. The lattice system is integrated using the Euler method with sample period $1 e-5$ running Matlab codes written by the authors. The parameters defining the dynamical system are selected the same for both the pdepe program and the Euler simulation. The initial states are assumed to be unity for both the pdepe and the Matlab code. The boundary condition is set to $q(t)=0.1 e^{0.5 t} \sin (t)$ with $\kappa_{0}=\kappa_{1}=0$. From Figure 1(b), the output responses of the original PDE system in (1) and the lattice system in (8) show good agreement when the number of discretisation points is large $(N=99)$. There is some mismatch between the corresponding responses when $N$ is relatively small $(N=4)$ as shown in Figure 1(a). The level of mismatch exhibited for the most extreme case, i.e. the case when the number of discretisation points is only 4 , is well within the bounds of uncertainty that can be dealt with effectively using a sliding mode approach.

\section{Step-by-step observer design for systems in lower Hessenberg form}

Consider the following system in lower Hessenberg form:

$$
\begin{aligned}
& \dot{x}(t)=A x(t)+\left[\begin{array}{c}
0 \\
\vdots \\
0 \\
a_{0}
\end{array}\right] q(t) \\
& y(t)=C x(t)=\left[\begin{array}{llll}
1 & 0 & \ldots & 0
\end{array}\right] x(t)
\end{aligned}
$$

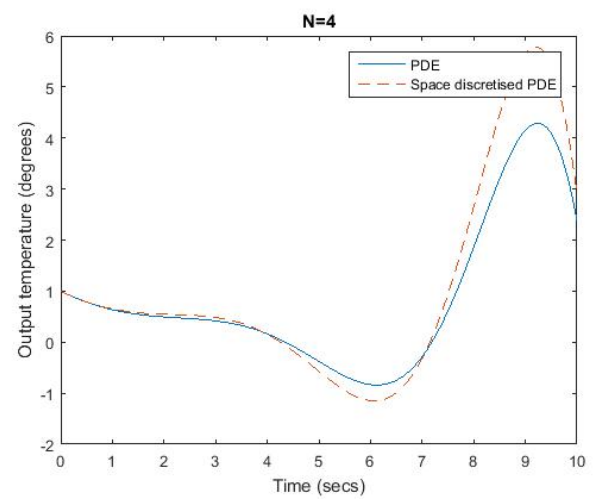

(a) $\mathrm{N}=4$

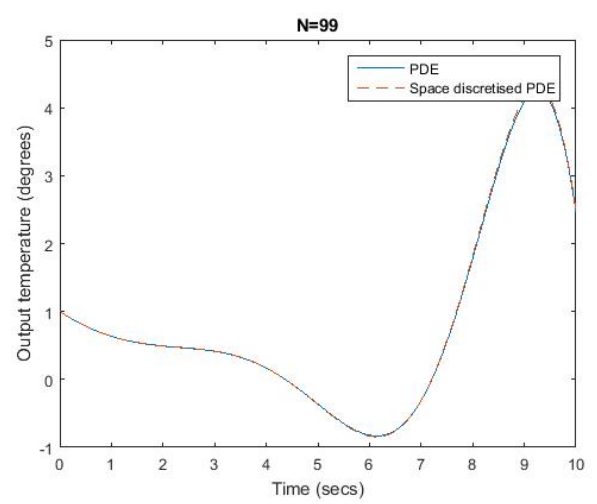

(b) $\mathrm{N}=99$

Fig. 1. Output responses of the lattice system discretised with the central difference scheme and the original PDE system 
Table 1. Values of $a_{i}$ for the system in (8)

\begin{tabular}{|c|c|c|c|c|c|}
\hline$a_{0}$ & $a_{1}$ & $a_{2}$ & $a_{3}$ & $a_{4}$ & $a_{5}$ \\
\hline$\frac{k_{1} \lambda_{1}}{h}+\frac{k_{3} \lambda_{1}}{2}$ & $-\frac{2 k_{1}}{h^{2}}+\frac{k_{1} \lambda_{0}}{h^{2}}+k_{2}-\frac{k_{3} \lambda_{0}}{2 h}$ & $\frac{k_{1}}{h^{2}}+\frac{k_{3}}{2 h}$ & $\frac{k_{1}}{h^{2}}-\frac{k_{3}}{2 h}$ & $-\frac{2 k_{1}}{h^{2}}+k_{2}$ & $-\frac{2 k_{1}}{h^{2}}+\frac{k_{1} \lambda_{1}}{h^{2}}+k_{2}+\frac{k_{3} \lambda_{1}}{2 h}$ \\
\hline
\end{tabular}

where the system matrix $A$ is given by

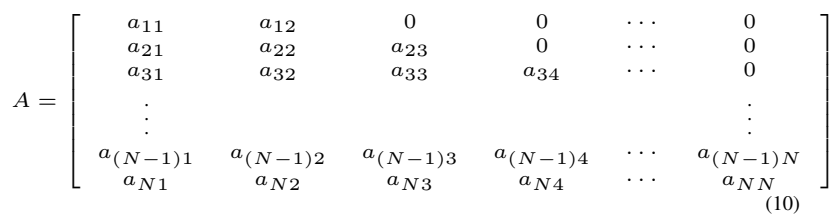

The tridiagonal Toeplitz-like form of (8) is a special case of this lower Hessenberg form. For system (9), define the step-by-step observer

$$
\dot{\widehat{x}}(t)=A \widehat{x}(t)+L C(x-\hat{x})+\left[\begin{array}{c}
\nu_{1}\left(\widetilde{x}_{1}-\widehat{x}_{1}\right) \\
\nu_{2}\left(\widetilde{x}_{2}-\widehat{x}_{2}\right) \\
\vdots \\
\nu_{N}\left(\widetilde{x}_{N}-\widehat{x}_{N}\right)
\end{array}\right]
$$

where $\widetilde{x}_{j}=\widehat{x}_{j}+\frac{1}{a_{(j-1) j}} \nu_{j-1, e q}\left(\widetilde{x}_{j-1}-\widehat{x}_{j-1}\right)$ and the output distribution matrix $C$ is as defined in (9). The terms $\nu_{j}\left(\widetilde{x}_{j}-\widehat{x}_{j}\right)$ are the observer injection terms which are to be designed so that the observer states converge to the system states in finite time. The associated terms $\nu_{j-1, e q}\left(\widetilde{x}_{j-1}-\widehat{x}_{j-1}\right)$ are the equivalent injection terms, which will be described later. The gain matrix $L=\left[l_{1}, l_{2}, \ldots, l_{N}\right]^{T}$ is a Luenberger observer gain selected to ensure $(A-L C)$ is stable. Initially $\widetilde{x}_{1}=x_{1}$ is defined. The observer (11) is a sliding mode observer, where as is usual [12], the sliding surface, $s$ is defined to be the output error

$$
s(t)=e_{1}=x_{1}-\widehat{x}_{1}=y-\widehat{y}
$$

The proof of convergence of the observer will now be developed.

\subsection{State and Unknown Input Estimation}

Let the state estimation error be $e=x(t)-\widehat{x}(t)$. Then the error dynamics is given by

$$
\dot{e}=(A-L C) e+\left[\begin{array}{c}
0 \\
\vdots \\
0 \\
a_{0}
\end{array}\right] q(t)-\left[\begin{array}{c}
\nu_{1}\left(\widetilde{x}_{1}-\widehat{x}_{1}\right) \\
\nu_{2}\left(\widetilde{x}_{2}-\widehat{x}_{2}\right) \\
\vdots \\
\nu_{N}\left(\widetilde{x}_{N}-\widehat{x}_{N}\right)
\end{array}\right]
$$

The objective of the step-by-step observer design is to make the errors go to zero successively, i.e., first $e_{1}=0$ is achieved, which brings about the condition needed to make $e_{2}=0$ and so on. This paradigm is widely exploited in the literature [3],[19],[12] and is now generalized for the lower Hessenberg form (13).

Theorem 1 If the observer injection terms are designed as

$$
\begin{aligned}
\nu_{i}\left(\widetilde{x}_{i}-\widehat{x}_{i}\right)= & K_{i} \operatorname{sign}\left(\widetilde{x}_{i}-\widehat{x}_{i}\right) \\
K_{1}> & \left(\left(\left|a_{11}\right|+\left|l_{1}\right|\right)\left|e_{1}\right|_{m}+\left|a_{12}\right|\left|e_{2}\right|_{m}\right) \\
K_{i}> & \left(\left|a_{i i}\right|\left|e_{i}\right|_{m}+\left|a_{i(i+1)}\right|\left|e_{i+1}\right|_{m}\right) \\
& i=2, \ldots, N \\
K_{N}> & \left(\left|a_{N N}\right|\left|e_{N}\right|_{m}+\left|a_{0}\right| U_{m}\right)
\end{aligned}
$$

where $\left|e_{i}\right|_{m}$ is the absolute maximum of the error $e_{i}(t)$ of the system (13) and $|q(t)|<U_{m} \forall t$, then the errors become zero in finite time in the sequence $i=$ $1,2, \ldots, N$, i.e., the estimated states of the observer (11) match the states of the system (9) in finite time and converge sequentially. Also, the unknown input $q(t)$ can be reconstructed once all the errors become zero.

Proof: By assumption, the absolute maximum values of each of the states are known, so the absolute maximum of the error is also known for each state. Denote these by $\left|e_{i}\right|_{m}$ where $i=\{1, \ldots, N\}$. The proof then proceeds stepwise as follows.

\section{Step 1:}

When $x_{1} \neq \widehat{x}_{1}$ (this is achieved by selecting $\widehat{x}_{1}(0) \neq$ $x_{1}(0)$ ), from the error dynamics (13), $\dot{e}_{1}$ sub-system becomes

$$
\dot{e}_{1}=\left(a_{11}+l_{1}\right) e_{1}+a_{12} e_{2}-K_{1} \operatorname{sign}\left(e_{1}\right)
$$

The well-known $\eta$-reachability condition [9] states that if

$$
e_{1} \dot{e}_{1}<-\eta\left\|e_{1}\right\|
$$

is satisfied, then $e_{1}$ will be identically zero after a finite time $t_{s} \leq \frac{\left|e_{1}(0)\right|}{\eta}$. It follows that with $K_{1}>\left(\left(\left|a_{11}\right|+\right.\right.$ $\left.\left.\left|l_{1}\right|\right)\left|e_{1}\right|_{m}+\left|a_{12}\right|\left|e_{2}\right|_{m}\right)$, a sliding mode occurs on $e_{1}=$ 0 in finite time.

The concept of the equivalent injection signal [9] is used to estimate the unmeasured signal $e_{2}$ while the 
system is sliding on $e_{1}=0$. The signal $\nu_{1, e q}$ represents the average behaviour of the discontinuous signal $\nu_{1}\left(e_{1}\right)$ and can be thought of as the injection signal required to maintain $e_{1}=0$, although the actual signal supplied to the system is the discontinuous signal $\nu_{1}$. This equivalent injection may be obtained by passing the discontinuous signal $\nu_{1}\left(\widetilde{x}_{1}-\widehat{x}_{1}\right)$ through a low pass filter. From (15)

$$
\dot{e}_{1}=\left(a_{11}+l_{1}\right) e_{1}+a_{12} e_{2}-\nu_{1, e q}\left(e_{1}\right)
$$

with $e_{1}=\dot{e}_{1}=0$ while sliding on the surface $e_{1}=0$. It follows from (17) that $e_{2}$ can be obtained as follows:

$$
e_{2}=\frac{1}{a_{12}} \nu_{1, e q}\left(e_{1}\right)
$$

Using (18), $x_{2}=\widehat{x}_{2}-\frac{1}{a_{12}} \nu_{1, e q}\left(\widetilde{x}_{1}-\widehat{x}_{1}\right)=\widetilde{x}_{2}$.

\section{Step 2 to $(\mathbf{N}-1)$ :}

As $e_{1}=0, x_{2}=\widetilde{x}_{2}$ and the correction term appearing in the dynamics of $e_{2}$ from the error dynamics becomes $\nu_{2}\left(e_{2}\right)=-K_{2} \operatorname{sign}\left(e_{2}\right)$ with $e_{2}$ given by (18). Thus when $e_{1}=0$

$$
\dot{e}_{2}=a_{22} e_{2}+a_{23} e_{3}-K_{2} \operatorname{sign}\left(e_{2}\right)
$$

With $K_{2}>\left(\left|a_{22}\right|\left|e_{2}\right|_{m}+\left|a_{23}\right|\left|e_{3}\right|_{m}\right)$, sliding will now take place on $e_{2}=0$ in finite time while maintaining the sliding on $e_{1}=0$. As before, during the sliding, it follows that

$$
e_{3}=\frac{1}{a_{23}} \nu_{2, e q}\left(e_{2}\right)
$$

Thus $x_{3}=\widehat{x}_{3}-\frac{1}{a_{23}} \nu_{2}\left(\widetilde{x}_{2}-\widehat{x}_{2}\right)=\widetilde{x}_{3}$. The correction term in the $e_{3}$ dynamics in the error dynamics (13) becomes $\nu_{3}\left(e_{3}\right)=-K_{3} \operatorname{sign}\left(e_{3}\right)$, with $e_{3}$ given by (20).

Proceeding in a similar way, one obtains $e_{i}=0$ successively in each step $(i=\{3, N-1\})$ by making $K_{i}>\left(\left|a_{i i}\right|\left|e_{i}\right|_{m}+\left|a_{i(i+1)}\right|\left|e_{i+1}\right|_{m}\right)$ with

$$
e_{i+1}=\frac{1}{a_{i(i+1)}} \nu_{i, e q}\left(e_{i}\right)
$$

\section{Step N:}

From the error dynamics (13) with $i=(N-1), e_{N}=$ $\frac{1}{a_{(N-1) N}} \nu_{N-1, e q}\left(e_{N-1}\right)$ and $x_{N}=\widetilde{x}_{N}$. The correction term appearing in the dynamics of $e_{N}$ in the error dynamics becomes $\nu_{N}\left(e_{N}\right)=-K_{N} \operatorname{sign}\left(e_{N}\right)$. The error dynamics is:

$$
\dot{e}_{N}=a_{N N} e_{N}+a_{0} q(t)-K_{N} \operatorname{sign}\left(e_{N}\right)
$$

With $K_{N}>\left(\left|a_{N N}\right|\left|e_{N}\right|_{m}+\left|a_{0}\right| U_{m}\right)$, one can ensure sliding takes place on $e_{N}=0$ in finite time. Thus all
Table 2. Simulation parameters for the observer (11) applied to the lattice System (8) without the unknown input

\begin{tabular}{|c|c|c|c|c|}
\hline $\mathrm{N}$ & $K_{1}$ & $m$ & $K_{N}$ & $\delta$ \\
\hline 4 & 10 & 0.1 & 12 & 0.0025 \\
\hline 99 & 10000 & 1000 & 60000 & 1 \\
\hline
\end{tabular}

the errors become zero sequentially and the system converges to the origin in finite time. When the system is at the origin, $e_{N}=0$ and $q(t)$ is given by

$$
q(t)=\frac{1}{a_{0}} \nu_{N, e q}\left(e_{N}\right)
$$

\subsection{Simulation - lattice system for stable PDE with} $k_{1}=1, k_{2}=-0.5$ and $k_{3}=-0.5$

Noting that the Lattice system (8) for the original PDE system (1) is a subclass of the Lower Hessenberg form, the developed observer design approach is applied. Discretisation using the central difference scheme is used to determine the corresponding lattice representation (8). Since the PDE is stable, the Luenberger gain matrix is set to zero. For the purpose of simulation, the switching terms are replaced by high gain feedback terms, with the exception of the final switching term, which is replaced by the sigmoid function $\left(\frac{\left|e_{N}\right|}{\left|e_{N}\right|+\delta}\right)$. This is justified as it is well-known that a discontinuous feedback is equivalent to highgain feedback [23]. The high gains can be implemented using the strategy found in [24]. The first gain $K_{1}$ is set and the successive gains are determined by the algorithm $K_{i+1}=\sqrt{m K_{i}}, i=\{1, N-2\}$ where $m$ is a design parameter. The gain $K_{N}$ is set separately. The initial states of the resulting $N$-th order ODE system are assumed to be all equal to unity, while the initial observer states are zero.

The performance of the proposed observer is first assessed without the unknown input. The simulation parameters are as in Table 2 and simulation results for $N=4$ and $N=99$ are presented in Figure 2. The estimation errors at the points of discretisation show the proposed sliding mode observer forces the errors to zero in finite time. The performance is now considered in the presence of the unknown input, considered as $q(t)=0.1 \sin 10 t$ for the purpose of simulation. Note that $q(t)$ is not known to the observer. The simulation parameters are presented in Table 3 and simulation results for $N=4$ and $N=99$ are presented in Figure 3 . It can be seen that in the presence of the unknown input, the quality of the state estimates at the points of discretisation degrade slightly. From Figure 4, the 


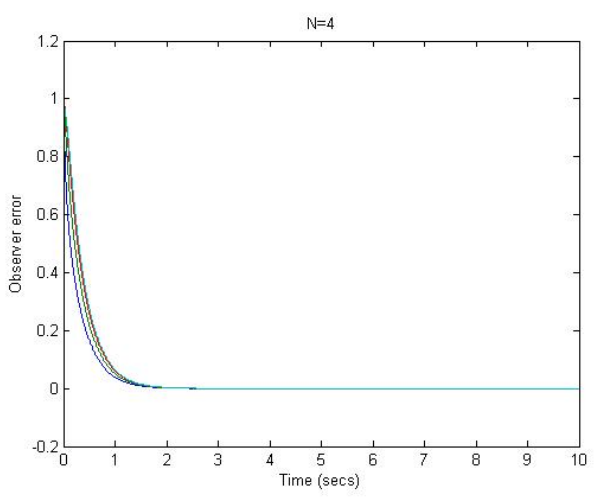

(a) $\mathrm{N}=4$

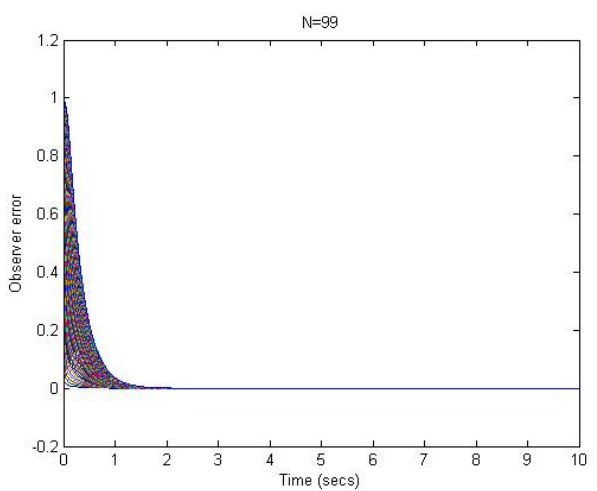

(b) $\mathrm{N}=99$

Fig. 2. Errors between the state of the lattice system (8) with $k_{1}=1$, $k_{2}=-0.5$ and $k_{3}=-0.5$ and observer (11) at the points of discretisation in the absence of an unknown input

Table 3. Simulation parameters for the observer (11) applied to the the lattice System (8) in the presence of the unknown input

\begin{tabular}{|c|c|c|c|c|}
\hline $\mathrm{N}$ & $K_{1}$ & $m$ & $K_{N}$ & $\delta$ \\
\hline 4 & 100 & 0.18 & 120 & 0.0025 \\
\hline 99 & 600000 & 10702 & 200000 & 0.7 \\
\hline
\end{tabular}

unknown input is reconstructed to a high degree of accuracy. The transient behaviour degrades for the case $N=99$ as may be expected from the fact that a large number of equivalent injection signals (21) have to converge to reach the sliding condition.

\section{Observer performance with the original PDE system}

The observer is now applied to estimate the states of the original PDE system (1) at the points of discretisation. Also, the unknown input $q(t)$ is estimated. The implementation of the observer is

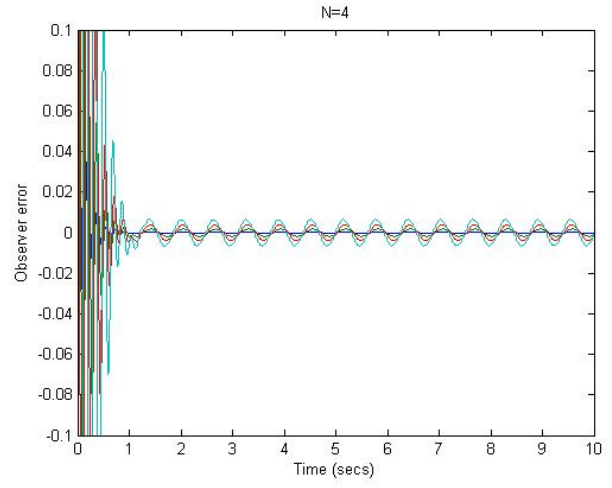

(a) $\mathrm{N}=4$

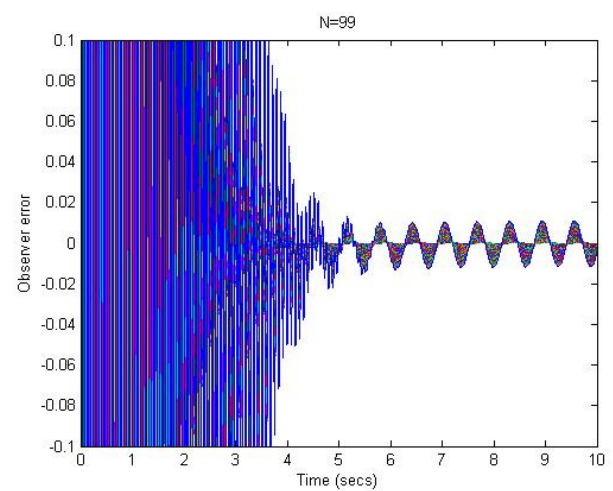

(b) $\mathrm{N}=99$

Fig. 3. Errors (zoom plot) between the state of the lattice system (8) with $k_{1}=1, k_{2}=-0.5$ and $k_{3}=-0.5$ and observer (11) at the points of discretisation with an unknown input

performed in the same way as discussed in subsection 3.2, except the errors are obtained by comparing the states from the actual PDE system, which is simulated using the pdepe program in Matlab [15]. The performance with both stable and unstable PDE systems is considered.

4.1. Case 1 - stable PDE with $k_{1}=1, k_{2}=-0.5$ and $k_{3}=-0.5$

The performance of the proposed observer is first assessed without the unknown input. As the PDE is stable, the Luenberger gain matrix is set to zero. The simulation parameters are as presented in Table 4 and simulation results for $N=4$ and $N=99$ are presented in Figure 5. The errors between the values of the actual states and the estimated states are presented at the points of discretisation and it is clear that the proposed sliding mode observer forces the errors to zero in finite time. It can be concluded that the proposed observer is 


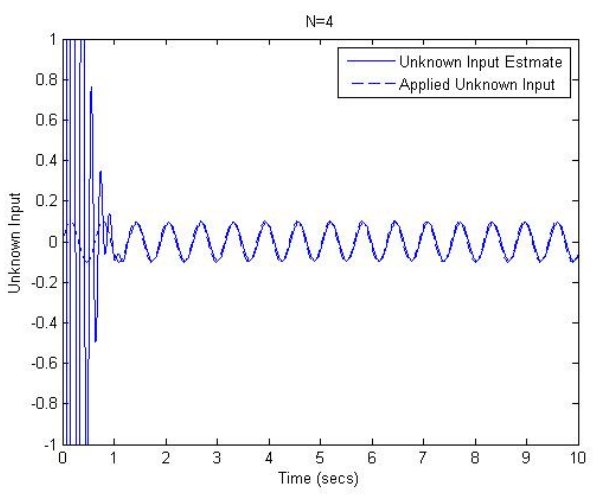

(a) $\mathrm{N}=4$

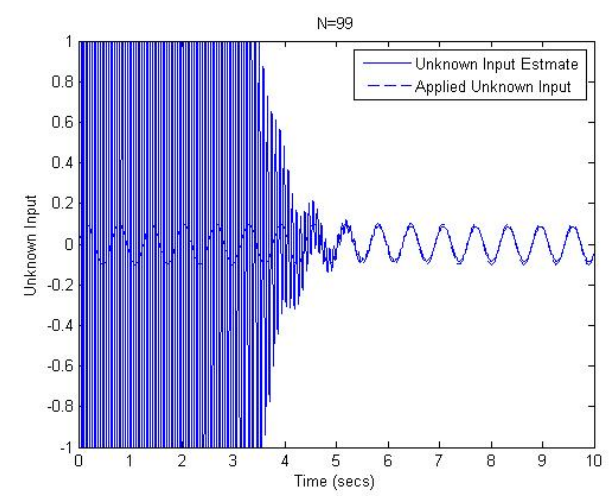

(b) $\mathrm{N}=99$

Fig. 4. Unknown Input Reconstruction for the lattice system (8) with $k_{1}=1, k_{2}=-0.5$ and $k_{3}=-0.5$

robust to the mismatch between the PDE (1) and the lattice system (8) in terms of estimation of the state at the points of discretisation. The errors are absorbed in the equivalent injection signals which represent, on average, the mismatch between the lattice system and the actual PDE system. The impact of increasing the number of discretisation points on the accuracy of the state estimates at the points of discretisation is negligible. The performance is now considered in the

Table 4. Simulation parameters for the observer (11) applied to the PDE system (1) with $k_{1}=1, k_{2}=-0.5$ and $k_{3}=-0.5$ without disturbances

\begin{tabular}{|c|c|c|c|c|}
\hline $\mathrm{N}$ & $K_{1}$ & $m$ & $K_{N}$ & $\delta$ \\
\hline 4 & 100 & 0.18 & 120 & 0.0025 \\
\hline 99 & 600000 & 10702 & 200000 & 0.7 \\
\hline
\end{tabular}

presence of the unknown input, considered as $q(t)=$ $0.1 \sin 10 t$ for the purpose of simulation. The simulation parameters are as presented in Table 5. Simulation results for $N=4$ are in Figure 6. Comparing Figure

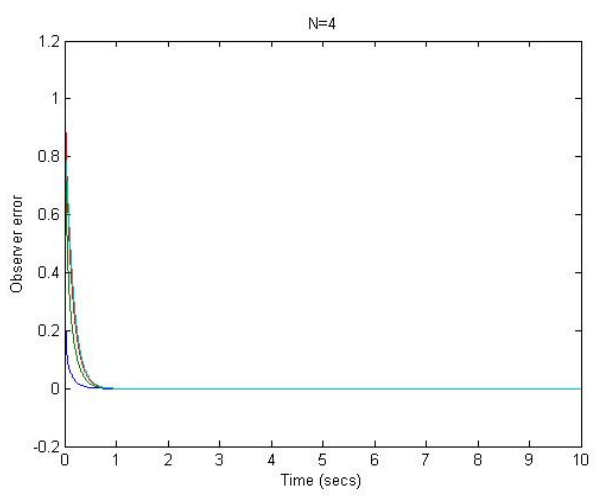

(a) $\mathrm{N}=4$

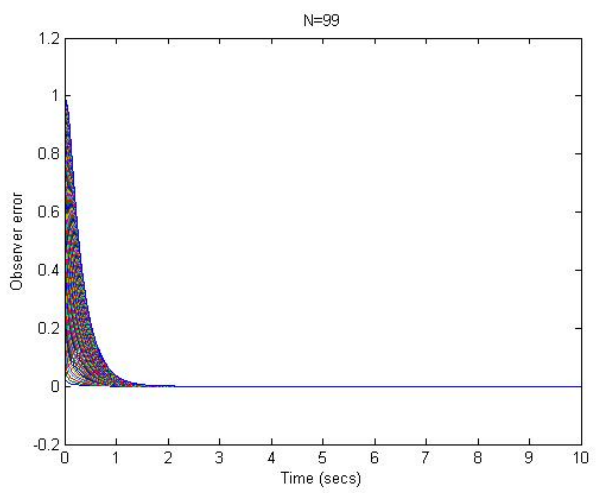

(b) $\mathrm{N}=99$

Fig. 5. Errors between the states at the points of discretisation for the PDE system (1) with $k_{1}=1, k_{2}=-0.5$ and $k_{3}=-0.5$ and observer (11) in the absence of an unknown input

3 and Figure 6 it is clear that even in presence of the unknown input, the estimation errors at the points of discretisation due to spacial discretisation of the PDE (1) are reasonable. The unknown input reconstruction shows some degradation in the fidelity of the estimate. It should be noted that the equivalent injection signals (21) are at every step counteracting mismatched disturbances (of order $O\left(h^{2}\right)$ ). This impacts on the performance of the unknown input estimation.

Table 5. Simulation parameters for the observer (11) applied to the PDE system (1) with $k_{1}=1, k_{2}=-0.5$ and $k_{3}=-0.5$ in the presence of an unknown input

\begin{tabular}{|c|c|c|c|c|}
\hline $\mathrm{N}$ & $K_{1}$ & $m$ & $K_{N}$ & $\delta$ \\
\hline 4 & 100 & 0.01 & 100 & 0.0025 \\
\hline
\end{tabular}


4.2. Case 2 - unstable PDE with $k_{1}=1, k_{2}=0.05$ and $k_{3}=-0.5$

To further test the applicability of the proposed observer to an unstable PDE plant (1), the reaction parameter $k_{2}$ is set to a positive constant and a corresponding observer (11) is designed. Choosing $k_{2}=0.05$ allows the instability to escape sufficiently slowly so that finite observation of the states can occur within an observation window of 0 to 10 seconds. The Luenberger gain matrix is parameterised so that the poles of $(A-L C)$ in (13) are specified to be as for the open-loop poles of the lattice system considered in Subsection 4.1. The performance of the proposed observer is first assessed without the unknown input. The simulation parameters are selected as for the stable PDE as presented in Table 4 and simulation results for $N=4$ and $N=99$ are presented in Figure 7. The errors between the values of the actual states and the estimated states are presented at the points of discretisation and the proposed sliding mode observer forces the errors

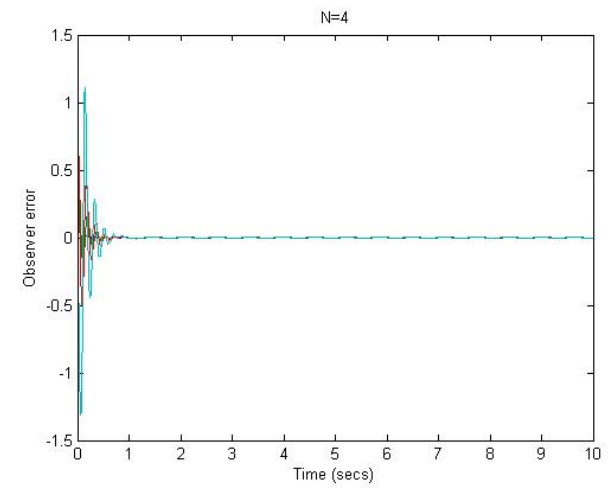

(a) Error plot

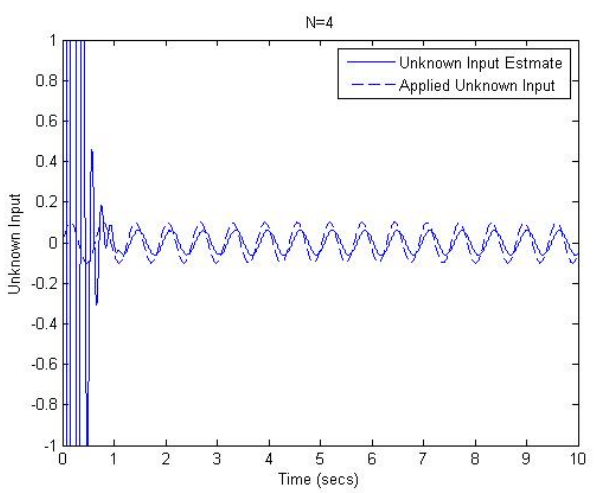

(b) Unknown input reconstruction

Fig. 6. PDE system (1) with $k_{1}=1, k_{2}=-0.5$ and $k_{3}=-0.5$ and observer (11) of order $\mathrm{N}=4$ in the presence of an unknown input

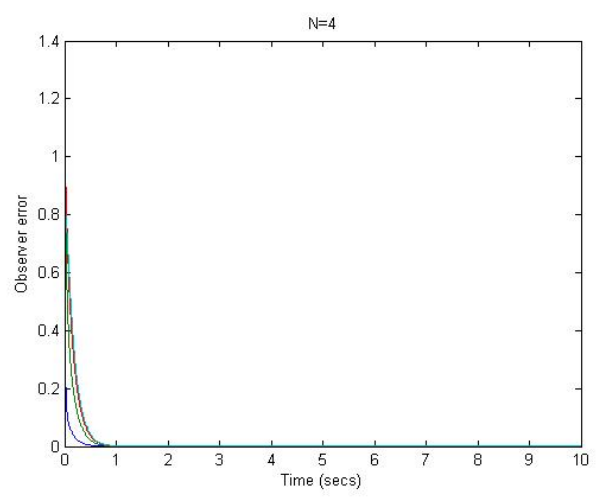

(a) $\mathrm{N}=4$

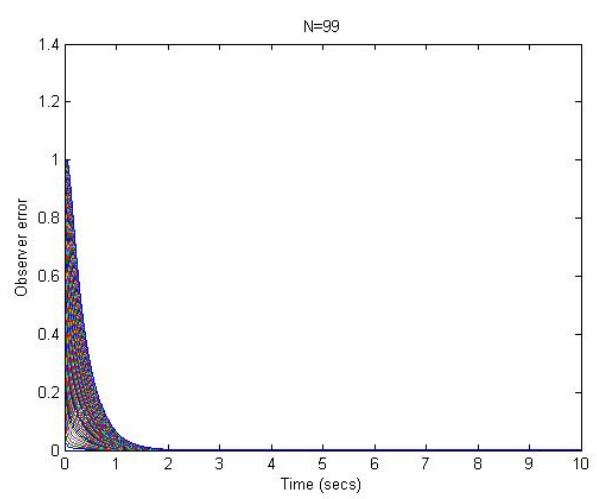

(b) $\mathrm{N}=99$

Fig. 7. Errors between the unstable PDE system with $k_{1}=1, k_{2}=$ 0.05 and $k_{3}=-0.5$ and observer in the absence of the unknown input

to zero in finite time even in the unstable case. The performance is now considered in the presence of the unknown input, considered as $q(t)=0.1 \sin 10 t$ for the purpose of simulation. The simulation parameters are as presented in Table 6. A zoomed plot of the errors between the values of the actual states and the estimated states is shown in Figure 8. The performance of the observer is not affected by the instability of the PDE system. The maximum magnitude of the error signals is consistent with that observed in the experiments presented for the stable case in Figure 6(a). The unknown input reconstruction is shown in Figure 9 where the degradation in the fidelity of the estimate is similar to that observed in the simulation testing of the stable PDE (Figure 6(b)).

\section{Conclusions}

A step-by-step sliding mode observer is designed for an ODE system in a lower Hessenberg form that 
Table 6. Simulation Parameters for Observer of Unstable PDE System with Disturbance

\begin{tabular}{|c|c|c|c|c|}
\hline $\mathrm{N}$ & $K_{1}$ & $m$ & $K_{N}$ & $\delta$ \\
\hline 4 & 100 & 0.2 & 100 & 0.0025 \\
\hline
\end{tabular}

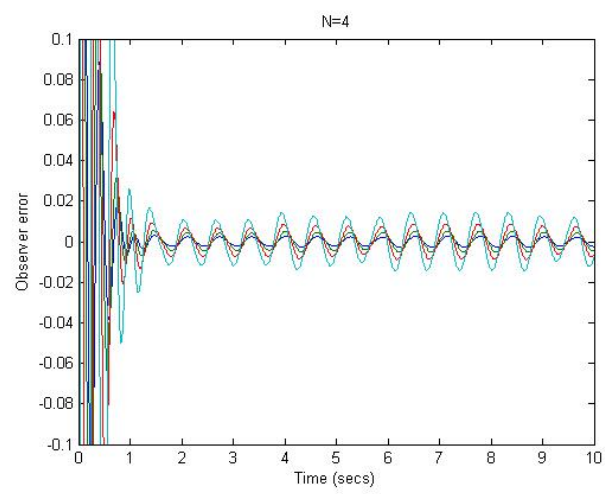

Fig. 8. Zoom of the errors between the unstable PDE system $k_{1}=1$, $k_{2}=0.05$ and $k_{3}=-0.5$ and observer of order $\mathrm{N}=4$ in the presence of the unknown input.

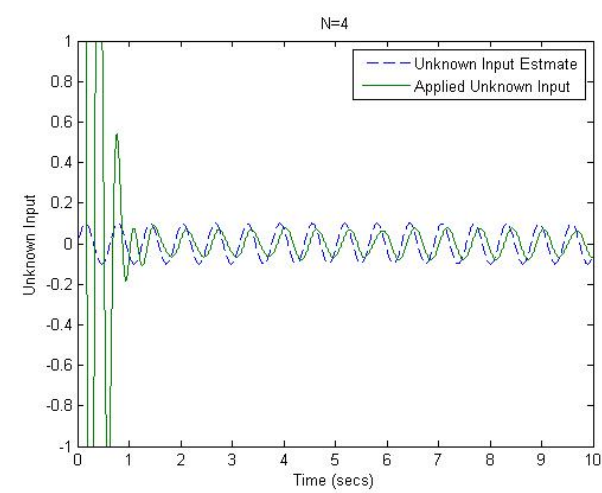

Fig. 9. Unknown Input Reconstruction for the unstable PDE system with the observer of order $\mathrm{N}=4$.

is shown to be particularly appropriate in the lattice setting. A reaction-advection-diffusion PDE is spatially discretised and approximated as an arbitrary finite order ODE system which is consistent with the structure of the proposed sliding mode observer. The observer is found to successfully estimate the states of the system at the spatial points of interest without having to build an infinite dimensional observer for the PDE system. The accuracy of the state estimates at the points of discretisation has been confirmed for both the lattice system and the original PDE system. It is also observed that estimation at a particular spatial point of interest does not depend on the discretisation order, as long as the point of interest is in the set of discretised points.
The observer can also track unknown inputs at one end of the spatial dimension when measurements are available at the other end. Perfect reconstruction is achieved for the lattice system and the property is independent of the chosen number of discretisation points. However, simulation studies have demonstrated that the accuracy of the estimate degrades when the observer is applied to the actual PDE system. This is expected as the equivalent injection signals represent, on average, the mismatch between the ODE system and the PDE system.

The proposed observer design, which is based on the approximation of the PDE by an appropriate ODE, appears to be universal in the sense that it is naturally in an implementable finite-dimensional form. The approach can be readily extended to higher spatial dimensions and to other PDE types.

The paper provides a framework for practical estimation of the states of a PDE system at particular spatial points and provides a mechanism to estimate any unknown input. Further work is needed to develop the design algorithm and this should be undertaken in tandem with study of significant practical applications such as the study of tumour growth.

\section{REFERENCES}

1. Ahmadizadeh, S., Zarei, J. \& Karimi, H. R. (2014). Robust Unknown Input Observer Design for Linear Uncertain Time Delay Systems with Application to Fault Detection. Asian J. Control, 16, (pp. 10061019).

2. Bamieh B.A., Paganini F. \& Dahleh M.A. (2002) Distributed control of spatially-invariant systems. IEEE Trans. Autom. Control, 47, (pp. 1091-1107).

3. Barbot, J. P., Boukhobza, T., \& Djemai, M. (1996). Sliding mode observer for triangular input form. Proc. 35th Conf. Decis. Control, Kobe, Japan, (pp. 1489-1490).

4. Chadli, M. \& Karimi, H.R. (2013). Robust Observer Design for Unknown Inputs TakagiSugeno Models. IEEE Trans. Fuzzy Systems, 21 (1), (pp. 158-164).

5. Chen, F., Zhang, K., Jiang, B. and Wen, C. (2016). Adaptive Sliding Mode Observer-Based Robust Fault Reconstruction for a Helicopter With Actuator Fault. Asian J. Control, 18 (4), (pp. 15581565).

6. Curtain, R. F, \& Zwart, H. J. (1995). An Introduction to Infinite-dimensional Linear Systems Theory, Springer-Verlag, New York.

7. Drakunov, S., \& Utkin, V. (1995). Sliding mode observers. Tutorial. Proc. 34th Conf. Conf. Decis. Control, New Orleans, LA, (pp. 3376-3378). 
8. Drakunov, S. V. (1995). Sliding-mode observers based on equivalent control method. Proc. 31st Conf. Decis. Control, Tuoson, Arizona, (pp. 23682369).

9. Edwards, C. \& Spurgeon, S. K. (1998). Sliding mode control: Theory \& Applications, Taylor \& Francis.

10. Edwards, C., Spurgeon, S. K., \& Patton, R. J. (2000). Sliding mode observers for fault detection and isolation. Automatica, 36 (4), (pp. 541-553).

11. Elhsoumi, A., Hadj Ali, S. B., Harabi, R. E. and Abdelkrim, M. N. (2016). Unknown Input Fault Detection and Isolation Observer Design for Neutral Systems. Asian J. Control, 18(5), (pp. 1934-6093).

12. Floquet, T., Edwards, C., \& Spurgeon, S. (2007). On sliding mode observers for systems with unknown inputs. Int. J. Adaptive Control \& Signal Processing, 21 (8-9), (pp. 638-656).

13. Guo, B. Z., \& Zhao, Z. L. (2015). Active Disturbance Rejection Control: Theoretical Perspectives. Communications in Information and Systems, 15 (3), (pp. 361 421).

14. Haskara, I. (1998). On sliding mode observers via equivalent control approach. Int. J. Control, 71 (6), (pp. 1051-1067).

15. Howard, P. (2005). Partial Differential Equations in MATLAB 7.0. www.math.tamu.edu/ phoward/m401/pdemat.pdf.

16. Krstic, M., \& Smyshlyaev, A. (2008). Boundary Control of PDEs: A Course on Backstepping Designs. SIAM Advances in Design \& ControL.

17. Newman, M. (2010) Networks: an Introduction. Oxford University Press.

18. Orlov, Y. (2009). Discontinuous Systems Lyapunov Analysis and Robust Synthesis under Uncertainty Conditions, Springer Verlag, Berlin.

19. Perruquetti, W., Floquet, T., \& Borne, P. (1998). A note on sliding observer and controller for generalized canonical forms. Proc. 37th Conf. Decis. Control, Tampa, Florida (pp. 1920-1925).

20. Smith, G. D. (1985). Numerical Solution of Partial Differential Equations: Finite Difference Methods. Clarendon Press.

21. Smyshlyaev, A., \& Krstic, M. (2010). Adaptive Control of Parabolic PDEs. Princeton University Press.

22. Spurgeon, S. K. (2008). Sliding mode observers: a survey. Int. J. Sys. Science, 39 (8), (pp. 751-764).

23. Utkin, V. (1977). Variable Structure Systems with Sliding Modes. IEEE Trans. Autom. Control, 22 (4), (pp. 212-222).
24. Utkin, V.I., Drakunov, S.V., Izosimov, D.B., Lukjanov, A.G, \& Utkin V.A. (1984). Hierarchical Principle of The Control System Decomposition Based on Motion Separation. Proc. 9th World IFAC Congress, Budapest, Hungary, (pp. 2-6).

25. Utkin, V.I.(1992) Sliding modes in Control Optimisation, Springer-Verlag, Berlin.

26. Wang, Y., Xia, Y., Shen, H. \& Zhou, P. (2018) SMC Design for Robust Stabilization of Nonlinear Markovian Jump Singular Systems. IEEE Trans. Autom. Control, 63 (1), (pp. 219-224).

27. Zhang, H., Zheng, X., Yan, H., Peng, C., Wang, Z. \& Chen Q. (2017) Codesign of Event-Triggered and Distributed $H_{\infty}$ Filtering for Active SemiVehicle Suspension Systems. IEEE/ASME Trans. Mechatronics, 22 (2), (pp. 1047-1058).

28. Zhao, Z., Liu, Y., He, W. \& Luo, F. (2016) Adaptive boundary control of an axially moving belt system with high acceleration/deceleration. IET Contr. Theory Appl, 10 (11), (pp. 1299-1306).

29. Zhao, Z., Liu, Z., Li, Z., Wang, N. \& Yang, J. (2017) Control design for a vibrating flexible marine riser system. J. Franklin Institute, 354 (18), (pp. 81178133).

30. Yan, H., Zhou, X., Zhang, H., Yang, F. \& Wu, Z.G. (2017) A Novel Sliding Mode Estimation for Microgrid Control with Communication Time Delays. IEEE Trans. Smart Grid, doi: 10.1109/TSG.2017.2771493.

31. Yang, J., Li, S. \& Yu, X. (2013) SlidingMode Control for Systems With Mismatched Uncertainties via a Disturbance Observer. IEEE Trans. Ind. Electronics, 60 (1), (pp. 160-169).

32. Yang, J., Li, S., Su, J. \& Yu, X. (2013) Continuous nonsingular terminal sliding mode control for systems with mismatched disturbances. Automatica, 49 (7), (pp. 2287-2291).

33. Yang, J., Su, J., Li, S. \& Yu, X. (2014) HighOrder Mismatched Disturbance Compensation for Motion Control Systems Via a Continuous Dynamic Sliding-Mode Approach. IEEE Trans. Ind. Informatics, 10 (1), (pp. 604-614). 